\title{
Médiévales
}

Langues, Textes, Histoire

51 | automne 2006

L'Occident sur ses marges ( $\left.\mathrm{V}\right|^{\mathrm{e}}-\mathrm{XI}^{\mathrm{e}}$ siècles)

\section{Constant Mews, La Voix d'Héloïse. Un dialogue de deux amants}

Paris, Fribourg, Cerf, Academic Press (Vestigia, $n^{\circ}$ 31), 2005, 347 p.

\section{Damien Boquet}

\section{OpenEdition}

\section{Journals}

Édition électronique

URL : https://journals.openedition.org/medievales/1458

DOI : 10.4000/medievales. 1458

ISSN : $1777-5892$

\section{Éditeur}

Presses universitaires de Vincennes

Édition imprimée

Date de publication : 1 décembre 2006

Pagination : 185-188

ISBN : 978-2-84292-193-4

ISSN : 0751-2708

\section{Référence électronique}

Damien Boquet, «Constant Mews, La Voix d'Héloïse. Un dialogue de deux amants », Médiévales [En ligne], 51 | automne 2006, mis en ligne le 12 mars 2007, consulté le 24 avril 2022. URL : http:// journals.openedition.org/medievales/1458; DOI : https://doi.org/10.4000/medievales.1458

Ce document a été généré automatiquement le 24 avril 2022.

Tous droits réservés 


\section{Constant Mews, La Voix d'Héloïse. Un dialogue de deux amants}

Paris, Fribourg, Cerf, Academic Press (Vestigia, n 31), 2005, 347 p.

Damien Boquet

1 Quoi de plus fidèle au mythe d'Héloïse et Abélard que la passion qui caractérise les débats récents autour des Epistolae duorum amantium (désormais EDA) ? Et pourtant, cette correspondance, issue d'une anthologie de lettres compilée à la fin du Xve siècle à Clairvaux par Johannes de Vepria (Troyes, BM, 1452, fo $159 \mathrm{r}^{\circ}-167 \mathrm{v}^{\circ}$ ) n'a guère électrisé la communauté des historiens lorsqu'Ewald Könsgen en a proposé l'édition critique en 1974, en évoquant déjà la possibilité d'une attribution aux célèbres amants des bords de Seine. Il s'agit d'un recueil de 113 lettres de longueur inégale, composées de messages et de quelques poèmes, dont beaucoup ont subi de fortes amputations dues au copiste. Philologue et amoureux du beau style, Johannes de Vepria (Jean de Woëvre) a sans doute écarté le contingent, toutes ces références concrètes qui aujourd'hui manquent cruellement pour certifier l'origine de ces lettres. Quoi qu'il en soit, deux voix s'expriment distinctement, celle d'un homme et celle d'une femme. Un couple d'amants qui demeure anonyme. Lui est un maitre réputé ; elle est son élève douée, «seule disciple de la philosophie parmi toutes les jeunes femmes de notre temps» (lettre 50), tous deux écrivent dans un style qui s'accorde avec la première moitié du XII siècle. On aurait pu penser alors que les historiens se seraient immédiatement emparés d'une telle découverte, pour confirmer ou infirmer l'hypothèse d'attribution. Eh bien non, ou plus exactement, comme l'explique Constant Mews, pendant 20 ans la braise a couvé sous la cendre. Lui-même, spécialiste de la théologie d'Abélard, ne s'est penché attentivement sur les EDA qu'en 1993, aussitôt « stupéfait à la lecture de certains mots et idées » qu'il savait appartenir précisément et exclusivement au lexique et à la pensée philosophiques d'Abélard. Depuis, il a mis entre parenthèses ses recherches sur Roscelin de Compiègne pour se consacrer aux EDA. Grand bien lui en a pris, car la parution des résultats de son enquête en 1999 dans un livre intitulé The Lost Love Letters of Heloise and Abelard - dont le présent ouvrage est la traduction française avec une postface inédite - fait événement à plus d'un titre. Tout d'abord, en plaidant « en faveur de la solution la plus simple, à savoir que les lettres ont bien été écrites par 
Abélard et Héloïse », l'A. a libéré un débat qui tardait à éclore. Mais, au-delà, il offre une mise en perspective neuve et suggestive des milieux lettrés en France du Nord au tournant des $\mathrm{XI}^{\mathrm{e}}$ et $\mathrm{XII}^{\mathrm{e}}$ siècles. Chemin faisant, l'A. prend un réel plaisir à écouter plus spécialement la voix Héloïse. Le titre français correspond ainsi mieux au contenu global de l'ouvrage car, derrière la singularité d'Héloïse, c'est bien le chœur d'une élite lettrée féminine que l'A. fait entendre.

2 Des six chapitres qui composent La Voix d'Héloïse, on peut tirer trois tableaux. Les deux premiers chapitres, en guise de prologue, guident le lecteur depuis la découverte du manuscrit par Könsgen et sa présentation minutieuse jusqu'à la tardive ouverture des débats sur l'attribution. La mise en lumière de la dramaturgie des lettres permet déjà de caractériser chacune des deux voix, qui se différencient très clairement dans leur conception respective de l'amour : lui parle d'un amor-passion qui se suffit à lui-même, elle développe une synthèse subtile de l'amicitia cicéronienne et de la dilectio chrétienne. On a là tout à la fois le cœur de leur dialogue amoureux et le point d'achoppement de la relation. L'A. profite de cette phase d'exposition pour dégager les rares références au monde extérieur, comme l'allusion à cet "entêtement français " (francigena cervicositas) que le maître doit affronter. Le second chapitre rappelle à quel point il est difficile d'approcher sereinement le couple mythique en raison de la complexité et de la profondeur de l'horizon d'attente qui se sont créées autour d'eux au fil des siècles. L'âpreté des débats dans les années 1970-1980 sur la correspondance monastique découverte par Jean de Meung, marqués par une forte tendance à minorer la parole d'Héloïse, explique selon l'A. que les EDA, publiées au même moment, aient été paradoxalement négligées. Qui pouvait vouloir d'une querelle d'authenticité supplémentaire? En tout cas, pas les derniers biographes d'Héloïse jusqu'à Guy Lobrichon qui, prenant le parti d'une "attribution indécidable " (Héloïse, l'amour et le savoir, Paris, Gallimard, 2005, p. 48), ne les intègre pas à son portrait, ce qui revient sur le plan historiographique précisément au même que d'opter pour la thèse de la non attribution.

3 Avec les chapitres 3 et 4 s'ouvre un nouveau tableau qui, sans jamais perdre de vue les $E D A$, offre une approche originale de la vie intellectuelle en France du Nord à la fin du $\mathrm{XI}^{\mathrm{e}}$ siècle-début du XII ${ }^{\mathrm{e}}$ siècle, plus précisément de la littérature amoureuse et du genre du dialogue homme-femme. L'A. montre alors que l'histoire racontée par les EDA n'est pas du tout incompatible avec l'Historia calamitatum, mais surtout qu'au travers du débat entre l'amour-passion (l'homme) et l'amitié-dilection (la femme), ce sont bien deux manières de promouvoir l'amour vrai qui s'expriment et cohabitent, avec d'une part la lignée unissant Guillaume de Champeaux, l'école de Saint-Victor et Bernard de Clairvaux à laquelle se rattacherait Héloïse alors que, d'autre part, les positions d'Abélard le rapprochent des milieux de la cour angevine et du camp des Garlande qui le protège. Les $E D A$ révèlent ainsi en contre-champ les enjeux culturels que portent ces différentes façons d'échafauder une éthique de l'amour. Par son étude du genre du dialogue amoureux dans le chapitre 4, l'A. rappelle que la voix d'Héloïse n'était pas sans écho féminin en son temps. Il y a bien sûr les figures féminines des corpus poétiques de Marbode de Rennes ou de Baudri de Bourgueil mais l'A. donne toute leur place à des correspondances moins connues, comme celles de Regensburg ou de Tegernsee au XII ${ }^{\mathrm{e}}$ siècle, qui exposent elles aussi des idéaux de relations homme/femme en termes de tension entre amor et amicitia. Quand le rideau tombe sur ce tableau, l'hypothèse de l'attribution se trouve renforcée d'une triple cohérence: cohérence des EDA avec 
l'Historia calamitatum; cohérence avec le genre du dialogue amoureux homme/femme ; cohérence avec le paysage culturel entre réforme et renaissance littéraire en France du Nord.

Le dernier tableau, avec les chapitres 5 et 6 , troque la longue vue de l'histoire culturelle pour le microscope de l'analyse textuelle. En l'absence d'éléments factuels, c'est bien dans la concordance du lexique qu'il faut se plonger prioritairement. Il conviendra, à terme, de procéder à une analyse systématique du vocabulaire des EDA, tâche lourde mais désormais possible à l'aide des bases de données informatisées. L'A. privilégie ici quelques pistes en variant les registres. Les résultats sont inégaux. Certains mots choisis sont trop peu spécifiques pour qu'on puisse en tirer des conclusions solides. C'est le cas par exemple de l'usage par la femme d'animus ou encore d'affectus. L'A. voit dans l'emploi fréquent de ce dernier mot un argument en faveur du rapprochement entre la conception de l'amour chez la femme et la rhétorique de Bernard de Clairvaux. Ce n'est pas moi qui vais remettre en cause la place centrale qu'occupe la notion d'affectus dans l'écriture cistercienne, mais le vocable est tellement commun à toute rhétorique affective (pas seulement cistercienne) qu'il est risqué d'en tirer des conclusions trop affirmatives. Les analyses consacrées au lexique philosophique sont les plus stimulantes. L'argument le plus probant concerne l'emploi par la femme du concept de scibilitas, la connaissabilité, un néologisme qui semble ne pas apparaître avant la fin du XIII ${ }^{\mathrm{e}}$ siècle (Raimond Lull) sauf chez... Abélard. On est aussi impressionné par la démonstration qui relie l'emploi d'indifferenter et de specialis/specialiter à la logique abélardienne. Ainsi, dans la lettre 24, l'homme ajoute à la conception cicéronienne de l'amitié qui fait de deux volontés distinctes une seule un "sans différence ». L'A. voit dans cet inattendu indifferenter une référence à la théorie des identités d'Abélard selon laquelle deux entités sans différence ne constituent pas pour autant une identité d'essence. En écho, le recours par la femme à l'adjectif specialis pour qualifier leur amour, alors que lui emploie singularis, serait une façon de sa part à elle de lui retourner son propre vocabulaire dialectique : par là, elle désignerait bien un amour " d'une espèce ", alors que lui en fait une res universalis. La lecture de l'A. sur ce point est virtuose mais néanmoins tout à fait probable tant les deux auteurs de la correspondance maîtrisent à un très haut niveau la joute intellectuelle dans leur vision respective de l'amour. Il convient malgré tout de reconnaitre que l'adverbe indifferenter n'est pas exceptionnel avec un sens plus commun (l'A. a raison de corriger P. von Moos qui prétend qu'indifferenter est un mot fréquent chez Aelred de Rievaulx ; en revanche, il n'est pas rare sous la plume de Bernard de Clairvaux et d'autres cisterciens contemporains); tout comme il n'y a rien a priori de bien spécifique au XII ${ }^{\mathrm{e}}$ siècle à voir un ami ou une amitié qualifiés de specialis, là encore avec un sens général de "privilégié(e)» (voir Bernard de Clairvaux, Pierre le Vénérable ou Aelred de Rievaulx). Le dernier chapitre tire les enseignements d'une attribution des EDA à Héloïse et Abélard, en revisitant la biographie croisée du couple et le paysage intellectuel de l'époque. L'A. expose de façon très convaincante comment Héloïse incarne la possibilité d'un compromis entre le courant cistercien et l'esprit philosophique abélardien.

5 Le livre s'achève sur une postface propre à la version française, dans laquelle l'A. répond à un certain nombre de critiques émises depuis la première édition de son livre en 1999, notamment par P. von Moos qui milite en faveur d'une datation tardive des $E D A$. Pour consolider sa thèse, l'A. apporte de nouveaux arguments. Celui qui consiste à rapprocher la conception de la caritas selon Abélard dans son œuvre savante avec la 
lettre 24 me paraît quelque peu forcé, tant la base est mince. En revanche, l'A. fait part d'une nouvelle trouvaille de poids: une allusion explicite à un passage peu cité de Cicéron (De amicitia, 20) dans la lettre 24 se trouve également dans la question $138 \mathrm{du}$ Sic et non.

6 Certes, une accumulation, même impressionnante, d'indices, de concordances textuelles et contextuelles ne vaut pas pour une preuve formelle. Mais c'est aussi justement au statut de la preuve en critique textuelle que ce véritable cas d'école invite à réfléchir. Au terme de la plaidoirie de l'A., une chose est certaine : il est plus difficile d'opter pour la thèse de la non attribution à Abélard et Héloïse que pour la thèse de l'attribution. D'autant plus difficile que d'autres éléments convergent dans le même sens (voir par exemple l'enquête de Sylvain Piron en postface de sa traduction des EDA chez Gallimard en 2005). Dès lors, la vraie question qui se pose désormais est : peut-on, pour peu que l'on reconnaisse que la vérité en histoire relève - ainsi que le rappelle avec bon sens S. Piron - d'une argumentation contradictoire, ne pas attribuer les EDA à Héloïse et Abélard ? En tout cas, il ne me paraît guère tenable pour l'historien de ne pas prendre position, sauf à rester sans voix sur Héloïse, sur sa relation avec Abélard, voire sur les cercles lettrés en France du Nord au début du XII siècle. 